ESAIM: PROCEEDINGS, December 2011, Vol. 34, p. 277-290

E. Cancès, V. Louvet and M. Massot, Editors

\title{
NEW RESOLUTION STRATEGY FOR MULTI-SCALE REACTION WAVES USING TIME OPERATOR SPLITTING AND SPACE ADAPTIVE MULTIRESOLUTION: APPLICATION TO HUMAN ISCHEMIC STROKE*
}

\author{
Max Duarte $^{1,2}$, Marc Massot $^{1}$, Stéphane Descombes $^{3}$, Christian Tenaud ${ }^{4}$, \\ Thierry Dumont ${ }^{5}$, Violaine Louvet $^{5}$ and Frédérique Laurent ${ }^{1}$
}

\begin{abstract}
We tackle the numerical simulation of reaction-diffusion equations modeling multi-scale reaction waves. This type of problems induces peculiar difficulties and potentially large stiffness which stem from the broad spectrum of temporal scales in the nonlinear chemical source term as well as from the presence of large spatial gradients in the reactive fronts, spatially very localized. A new resolution strategy was recently introduced [11] that combines a performing time operator splitting with high oder dedicated time integration methods and space adaptive multiresolution. Based on recent theoretical studies of numerical analysis, such a strategy leads to a splitting time step which is not restricted neither by the fastest scales in the source term nor by stability limits related to the diffusion problem, but only by the physics of the phenomenon. In this paper, the efficiency of the method is evaluated through 2D and 3D numerical simulations of a human ischemic stroke model, conducted on a simplified brain geometry, for which a simple parallelization strategy for shared memory architectures was implemented, in order to reduce computing costs related to "detailed chemistry" features of the model.
\end{abstract}

\section{INTRODUCTION}

Numerical simulations of multi-scale phenomena are commonly used for modeling purposes in many applications such as combustion, chemical vapor deposition, or air pollution modeling. In general, all these models raise several difficulties created by the large number of unknowns and the wide range of temporal scales due to large and detailed chemical kinetic mechanisms, as well as steep spatial gradients associated with very localized fronts of high chemical activity. To overcome these difficulties, dedicated numerical methods were developed

* This research was supported by a fundamental project grant from ANR (French National Research Agency - ANR Blancs) Séchelles (project leader S. Descombes - 2009-2013), by a CNRS PEPS Maths-ST2I project MIPAC (project leader V. Louvet - 2009-2010), and by a DIGITEO RTRA project MUSE (project leader M. Massot - 2010-2014).

${ }^{1}$ Laboratoire EM2C - UPR CNRS 288, Ecole Centrale Paris, Grande Voie des Vignes, 92295 Chatenay-Malabry Cedex, France; e-mail: max.duarte@em2c.ecp.fr, marc.massot@em2c.ecp.fr,frederique.laurent@em2c.ecp.fr

2 Ph.D. grant from Mathematics (INSMI) and Engineering (INSIS) Institutes of CNRS and supported by INCA project (CNRS/ONERA/SAFRAN).

${ }^{3}$ Laboratoire J. A. Dieudonné - UMR CNRS 6621, Université de Nice - Sophia Antipolis, Parc Valrose, 06108 Nice Cedex 02 , France; e-mail: sdescomb@unice.fr

${ }^{4}$ LIMSI - CNRS, B.P. 133, Campus d'Orsay, 91403 Orsay Cedex, France; e-mail: tenaud@limsi.fr

${ }^{5}$ Institut Camille Jordan - UMR CNRS 5208, Université de Lyon, Université Lyon 1, INSA de Lyon 69621, Ecole Centrale de

Lyon, 43 Boulevard du 11 novembre 1918, 69622 Villeurbanne Cedex, France; e-mail: tdumont@math.univ-lyon1.fr, louvet@math.univ-lyon1.fr

(C) EDP Sciences, SMAI 2011 
to solve these strongly coupled nonlinear systems with either fully implicit, semi-implicit or yet linearized implicit methods (see [6] and references therein). Nevertheless, the computing cost and the important memory requirements of these methods, even if adaptive grids are used, make these strategies difficult to be handled.

In order to reduce these computational requirements, a family of methods called IMEX, wisely combines implicit and explicit time discretization to solve stiff nonlinear problems [16,20]. Even though these methods are usually very efficient, a natural drawback is that the time steps globally imposed over partial regions or the entire domain are strongly limited by either the stability restrictions of the explicit solver or by the fastest scales treated by the implicit scheme. We know though that these fastest time scales do not always play a leading role in the global physics of many multi-scale problems. Therefore, one might consider using reduced models where these chemical scales have been previously relaxed, with a significant reduction of computing costs. Nevertheless, these solutions reproduce only approximate dynamics and are usually accessible when the system is well-partitioned or the fastest scales can be identified or isolated, a process often hard to conduct and justify in realistic configurations.

Since the resolution of the fully coupled problem is most of the time out of reach and the appropriate definition of reduced models is usually difficult to establish, time operator splitting methods have been used for a long time and there exists a large literature showing the efficiency of such methods for evolution problems. A splitting procedure allows to consider dedicated solvers for the reaction part which is decoupled from the other physical phenomena like convection, diffusion or both, for which there also exist dedicated numerical methods. Hence, a completely independent optimization of the resolution of each subsystem might be pursued. In order to guarantee the accuracy of the solution obtained by a splitting scheme, the splitting time steps used for the independent resolution of each subproblem are usually taken of the order of the fastest scales included in the problem. As a matter of fact, several works $[6,17,21]$ showed that the standard numerical analysis of splitting schemes fails in presence of scales much faster than the splitting time step.

More rigorous studies for time/space stiff configurations were then conducted that allow to better describe the influence of stiffness on the numerical behavior of splitting methods and demonstrate that the error of integration is given by the splitting time step even if the latter is larger than any fast scale of the problem and even if there is a loss of order of the splitting schemes [7-9]. As a consequence, a new time operator splitting approach was introduced [11] where the global solution is reconstructed by a splitting scheme with splitting time steps dictated by the global physical coupling, possibly much larger than the fastest time scales, whereas the dedicated methods used to solve each subsystem are responsible for dealing with the fast scales associated with each one of them, in a separate manner. In this way, the splitting time step is chosen based only on error estimates of the numerical simulation in order to guarantee the description of the physics of the phenomenon within a prescribed accuracy, without being related to the stability constraints of the numerical resolution of each subsystem and with an important improvement of efficiency whenever a broad decoupling of the time scale spectrum is possible.

This time integration strategy have been successfully applied to 2D and 3D simulations of a stiff reactiondiffusion system modeling human ischemic strokes with a 19-species detailed chemistry [10] in a realistic brain geometry [12]. In this paper, the same model is solved on a simplified brain geometry with a mesh refinement technique based on adaptive multiresolution methods [5,15] in order to improve the performance of the splitting scheme [11]. In particular, we extend the domain of application of the numerical strategy proposed in [11] to stiffer and more complex configurations. Exploiting the splitting configuration, a parallel computing technique was also implemented for the time integration stage in the framework of shared memory computing architectures. Some preliminary results were already shown in [12] in another context without any detail or analysis of the numerical performance of the method. The paper is organized as follows: In Section 2, we recall the main aspects of the numerical strategy introduced in [11]. 2D and 3D simulations of a human ischemic stroke model [10] are presented in Section 3 to illustrate the potential and performance of the method. We end in Section 4 with some concluding remarks and prospects. 


\section{Numerical Strategy}

In this section, we detail briefly the main elements of the numerical strategy introduced in [11] for the resolution of multi-scale reaction waves.

\subsection{Time Operator Splitting}

Let us first set the general mathematical framework in this work. A class of multi-scale phenomena are modeled by general reaction-diffusion systems like:

$$
\left.\begin{array}{ll}
\partial_{t} \mathbf{u}-\partial_{\mathbf{x}}\left(\mathbf{D}(\mathbf{u}) \partial_{\mathbf{x}} \mathbf{u}\right)=\mathbf{f}(\mathbf{u}), & \mathbf{x} \in \mathbb{R}^{d}, t>0, \\
\mathbf{u}(0, \mathbf{x})=\mathbf{u}_{0}(\mathbf{x}), & \mathbf{x} \in \mathbb{R}^{d},
\end{array}\right\}
$$

where $\mathbf{f}: \mathbb{R}^{m} \rightarrow \mathbb{R}^{m}$ and $\mathbf{u}: \mathbb{R} \times \mathbb{R}^{d} \rightarrow \mathbb{R}^{m}$, with the diffusion matrix $\mathbf{D}(\mathbf{u})$, which is a tensor of order $d \times d \times m$.

In order to simplify the presentation, we consider problem (1) with linear diagonal diffusion, in which case the elements of the diffusion matrix are written as $D_{i j k}(\mathbf{u})=D_{k} \delta_{i j}$, so that the diffusion operator reduces to the heat operator with scalar diffusion coefficient $D_{k}$ for component $u_{k}$ of $\mathbf{u}, k=1, \ldots, m$. In any case, the numerical strategy proposed in [11] can handle the general problem (1). Performing a fine spatial discretization, we obtain the semi-discretized initial value problem:

$$
\left.\begin{array}{l}
\mathrm{d}_{t} \mathbf{U}-\mathbf{B} \mathbf{U}=\mathbf{F}(\mathbf{U}), \quad t>0, \\
\mathbf{U}(0)=\mathbf{U}^{0},
\end{array}\right\}
$$

where $\mathbf{B}$ corresponds to the discretization of the Laplacian operator with the coefficients $D_{k}$ within. $\mathbf{U}$ and $\mathbf{F}(\mathbf{U})$ are arranged component-wise all over the discretized spatial domain. Considering a standard decoupling of the diffusion and reaction parts of (2), we denote $\mathcal{X}^{\Delta t}\left(\mathbf{U}^{0}\right)$ as the numerical solution of the discretized diffusion equation:

$$
\mathrm{d}_{t} \mathbf{U}_{D}-\mathbf{B} \mathbf{U}_{D}=0, \quad t>0
$$

with initial data $\mathbf{U}_{D}(0)=\mathbf{U}^{0}$ after an integration time step $\Delta t$. We also denote by $\mathcal{Y}^{\Delta t}\left(\mathbf{U}^{0}\right)$ the numerical solution of the reaction part:

with initial data $\mathbf{U}_{R}(0)=\mathbf{U}^{0}$.

$$
\mathrm{d}_{t} \mathbf{U}_{R}=\mathbf{F}\left(\mathbf{U}_{R}\right), \quad t>0,
$$

The two Lie approximation formulae of the solution of system (2) are then defined by

$$
\mathcal{L}_{1}^{\Delta t}\left(\mathbf{U}^{0}\right)=\mathcal{X}^{\Delta t} \mathcal{Y}^{\Delta t}\left(\mathbf{U}^{0}\right), \quad \mathcal{L}_{2}^{\Delta t}\left(\mathbf{U}^{0}\right)=\mathcal{Y}^{\Delta t} \mathcal{X}^{\Delta t}\left(\mathbf{U}^{0}\right),
$$

whereas the two Strang approximation formulae [18] are given by

$$
\mathcal{S}_{1}^{\Delta t}\left(\mathbf{U}^{0}\right)=\mathcal{X}^{\Delta t / 2} \mathcal{Y}^{\Delta t} \mathcal{X}^{\Delta t / 2}\left(\mathbf{U}^{0}\right), \quad \mathcal{S}_{2}^{\Delta t}\left(\mathbf{U}^{0}\right)=\mathcal{Y}^{\Delta t / 2} \mathcal{X}^{\Delta t} \mathcal{Y}^{\Delta t / 2}\left(\mathbf{U}^{0}\right),
$$

where $\Delta t$ is now the splitting time step. It is well known that Lie formulae (5) (resp. Strang formulae (6)) are approximations of order 1 (resp. 2) of the exact solution of (2) in the case where $\mathcal{X}^{\Delta t}$ and $\mathcal{Y}^{\Delta t}$ are the exact solutions $X^{\Delta t}$ and $Y^{\Delta t}$ of problems (3) and (4).

\subsection{Time Integration Strategy}

The standard orders achieved with a Lie or Strang scheme are no longer valid when we consider very stiff reactive or diffusive terms (see [9]). Furthermore, if the fastest time scales play a leading role in the global physics of the phenomenon, then the solution obtained by means of a splitting composition scheme will surely 
fail to capture the global dynamics of the phenomenon, unless we consider splitting time steps small enough to solve such scales.

In the opposite case where the fastest scales are not directly related to the physical evolution of the phenomenon, larger splitting time steps might be considered, but order reductions may then appear due to short-life transients associated with the fast variables. This is usually the case for propagating reaction waves where for instance, the speed of propagation is much slower than the chemical scales. In this context, it has been proved in [9] that better performances are expected while ending the splitting scheme by the time integration of the reaction part (4) or in a more general case, the part involving the fastest time scales of the phenomenon (see a numerical case in [8]). In particular, in the case of stiff reaction-diffusion systems with linear diagonal diffusion, no order loss is expected for the $\mathcal{L}_{2}^{\Delta t}$ and $\mathcal{S}_{2}^{\Delta t}$ schemes when faster scales are present in the reactive term. However, one must also take into account possible order reductions coming this time from space multi-scale phenomena due to steep spatial gradients whenever large splitting time steps are considered, as analyzed in [7].

These theoretical studies allow to depict more precisely the numerical behavior of the splitting techniques and thus, help us to select among the various splitting alternatives, depending on the nature of the problem. Nevertheless, the choice of suitable time integration methods for each subsystem is mandatory not only to guarantee such theoretical estimates but also to take advantage of the particular features of each independent subproblem in order to solve them with reasonable resources as accurately as possible. In particular, our splitting technique considers high order dedicated integration methods for each subproblem in order to properly solve the fastest time scales associated with each one of them, and in such a way that the main source of error is led by the operator splitting error. Then, the control of the accuracy of the time integration is ruled by the splitting scheme by means of the splitting time step needed to describe the global physical phenomenon within a required level of accuracy.

\subsubsection{Time Integration of the Reaction: Radau5.}

Radau5 [13] is a fifth order implicit Runge-Kutta method that guarantees $A$ - and $L$-stability properties, so that very stiff systems of ODEs might be solved without any stability problem. Even though nonlinear systems must be solved throughout the time integration process and important memory requirements are consequently needed, we easily overcome this difficulty in a splitting scheme context because the reactive term of (2) is a system of ODEs without spatial coupling. A local approach node by node is therefore adopted where the memory requirements are only set by the number of local unknowns, which usually does not exceed conventional memory resources. Even more, this approach is posed as an embarrassingly parallel problem in which each core solves successively one single node where no data exchange is needed among nodes, and therefore yields optimal load balancing on shared memory architectures.

Radau5 features also an adaptive time stepping strategy which guarantees a requested accuracy of the numerical integration. An important gain of efficiency is achieved in a splitting context because the reaction time integration step $\Delta t_{R}$ will be adapted only at nodes with high reactive activity in the neighborhood of the wavefront. Consequently, local reaction time steps much smaller than the splitting time step will be only partially needed, while the global time advancement of the resolution given by the splitting time step $\Delta t$ will not be limited by these fast time scales.

\subsubsection{Time Integration of the Diffusion: ROCK4.}

ROCK4 [1] is a fourth order stabilized explicit Runge-Kutta method and such methods feature extended stability domain along the negative real axis [19]. The method is then very appropriate for diffusion problems because of the usual predominance of negative real eigenvalues for which the method is efficiently stable. A very suitable example is the linear diagonal diffusion problem (3) with only negative real eigenvalues and constant spectral radius $\rho(\mathbf{B})$. An important gain of efficiency is obtained in this case because the discretized diffusion operator has a sparse matrix structure that yields more performing matrix-vector products during the time integration process. Furthermore, the resolution of diffusion problem (3) can be parallelized so that each core solves successively one single diffusion problem for only one specie, yielding an important reduction in the number of variables and consequently, in CPU time. 
ROCK4 features also dynamic time step adaptation so that the diffusion time integration step $\Delta t_{D}$ necessary to guarantee a prescribed accuracy, may be much smaller than the splitting time step $\Delta t$. Considering a general problem such as $\mathbf{v}^{\prime}=\mathbf{g}(\mathbf{v})$, in order to guarantee the stability of computations for a given time step $\Delta t_{D}$, the number of stages $s$ needed is directly related to the spectral radius $\rho(\partial \mathbf{g} / \partial \mathbf{v})$, as long as the latter is dominated by real negative eigenvalues. Hence, for a given $\Delta t_{D}$ needed to guarantee the accuracy of the integration, the minimum number of stages $s$ needed to guarantee the stability is computed by the ROCK4 solver through

$$
0.35 \cdot s^{2} \geq \Delta t_{D} \rho\left(\frac{\partial \mathbf{g}}{\partial \mathbf{v}}(\mathbf{v})\right)
$$

which extends quadratically on $s$ the stability domain of the method along the negative axis.

\subsection{Mesh Refinement Technique}

In the context of propagating wavefronts, important reactive activity as well as steep spatial gradients are localized phenomena. This implies that if we consider the resolution of reactive problem (4), a considerable amount of computing time is spent on nodes that are practically at (partial) equilibrium. Moreover, there is no need to represent these quasi-stationary regions with the same spatial discretization needed to describe the reacting wavefront, so that the diffusion problem (3) might also be solved over a smaller number of nodes. Therefore, an adaptive mesh refinement technique obtained by a multiresolution analysis [4, 15] was coupled with the latter time operator splitting technique in [11] for the resolution of multi-scale reaction waves.

Considering a set of nested spatial grids $j$ with $j=0,1, \cdots, \mathrm{J}$ from the coarsest to the finest one, a multiresolution transformation allows to represent a discretized function as values on a coarser grid plus a series of local estimates at different levels of such nested grids. These estimates correspond to the wavelet coefficients of a wavelet decomposition obtained by inter-level operations, and retain the information on local regularity when going from a coarse to a finer grid. Hence, lower wavelet coefficients are associated with local regular spatial configurations and vice-versa. A thresholding process applied to this representation allows finally to dynamically build the corresponding adapted grid on which the solutions are represented $[5,15]$.

Based on the mathematical basis of the multiresolution analysis, an error of the spatial representation of the solution represented on the adapted grid can be inferred with respect to the solution discretized on the finest grid. Hence, the error introduced by the multiresolution transformation is proportional to $\varepsilon$, where $\varepsilon$ is a threshold parameter [5,14]. Finally, this error estimate of the compressed spatial representation as well as the numerical behavior of the time operator splitting schemes, allow one to properly choose the simulation parameters in order to predict the expected level of accuracy of the resolution [11].

\subsection{Choice of the Splitting Time Step}

In the case of propagating wavefronts, we have shown in [11] that a constant splitting time step is good enough to capture the dynamics of the phenomenon. This splitting time step is set by the desired level of accuracy in the resolution of the wave speed, the wave profile, both, or any other parameter, depending on the problem and considering that each subsystem if perfectly solved. It is thus only depending on the phenomenon we want to describe and therefore, on the degree of decoupling we can achieve between the various subsystems within a prescribed error tolerance. In this particular application, we have chosen a splitting time step $\Delta t$ that verifies:

$$
E_{v}=\frac{\left|v-v_{s p l i t}\right|}{v} \leq \eta_{v}
$$

where $\eta_{v}$ is an accuracy tolerance for the velocity error $E_{v}$, considering a reference wave solution $\mathbf{U}$ of semidiscretized problem (2) with corresponding wavefront speed $v$, and the approximated solution $\mathbf{U}_{\text {split }}$ of speed $v_{\text {split }}$, computed by the operator splitting technique. To guarantee an accurate resolution of the reaction and diffusion problems, the corresponding accuracy tolerances $\eta_{R a d a u}$ and $\eta_{R O C K 4}$ of these solvers must verify:

$$
\eta_{\text {Radau } 5}, \eta_{R O C K 4}<\eta_{v} .
$$


In this way, we can isolate the various integration errors and guarantee that the overall time integration error is practically given by the splitting scheme approximation.

Taking into account that the time evolution is performed on an adapted grid, fixed during each time step, the propagating locally refined spatial gradients should remain into the corresponding fine regions during each time step evolution $\Delta t$. In order to guarantee this for a given spatial discretization $(\Delta x, \Delta y, \Delta z)$ corresponding to the finest $\mathrm{J}$ level, the splitting time step must be bounded by a maximum splitting time step $\Delta t_{\max }$ computed by:

$$
\Delta t \leq \Delta t_{\max }=\min \left\{\frac{\hat{k} \Delta x}{v_{x}}, \frac{\hat{k} \Delta y}{v_{y}}, \frac{\hat{k} \Delta z}{v_{z}}\right\},
$$

for the directional components $\left(v_{x}, v_{y}, v_{z}\right)$ of the wavefront speed and where $\hat{k} \geq 2$ stands for the refined region obtained with a standard refinement criteria detailed in [11].

\section{Numerical Simulations}

In this last section, we present some numerical illustrations of the proposed strategy. The performance of the method is discussed in the context of $2 \mathrm{D}$ and $3 \mathrm{D}$ simulations of a human ischemic stroke model which is briefly presented in what follows.

\subsection{Ischemic Stroke Model}

The model is based on a reaction-diffusion system of type (2) and considers ionic movements, glutamate excitotoxicity, cytotoxic edema and spreading depressions [10]. It focuses on the first hour of a stroke, when the ionic exchanges are the main mechanisms leading to cell death. Brain tissue is composed of two cell types, namely neurons and glial cells, and of extracellular space. In general, two domains are considered: the white and the grey matter which differ in their glial cell composition (astrocytes in grey matter and oligodendrocytes in white matter), and in their neuronal area composition (neuronal somas in grey matter and neuronal axons in white matter); nevertheless, in these simulations only grey matter will be taken into account. The ionic species considered are $\mathrm{K}^{+}, \mathrm{Na}^{+}, \mathrm{Cl}^{-}, \mathrm{Ca}^{2+}$ and the Glutamate $(g l u)$. They pass through neuronal and glial membranes via ionic channels (such as voltage-gated channels, receptor-channels, stretch-channels) and via ionic pumps and transporters (which are energy-dependent). Finally, the model considers the following variables, dependent from both coordinates and time:

(1) Volume fractions $f_{n}$ and $f_{a}$ (by brain volume unit) of neurons and glial cells. Extracellular volume fraction is thus given by $1-f_{n}-f_{a}$.

(2) Membrane potentials $V_{n}$ and $V_{a}$ of neurons and glial cells.

(3) Concentrations of $\mathrm{K}^{+}, \mathrm{Na}^{+}, \mathrm{Cl}^{-}, \mathrm{Ca}^{2+}$ and $\mathrm{glu}$ for neurons, glial cells and extracellular space.

Consequently, one has to solve a reaction-diffusion system with $m=19$ unknowns, noticing that there is no diffusion for 9 variables: $f_{n}, f_{a}, V_{n}, V_{a}$ and ions in neurons. There are no fluxes of ions in and out of the brain and thus, the boundary conditions are of Neumann homogeneous type. A stable equilibrium solution is taken as initial condition $\mathbf{U}(0)$. For the moment, only simplified geometries can be simulated with the adaptive mesh refinement technique (see [12] for simulations on realistic brain geometries).

Let us finally remark that the reaction term $\mathbf{F}(\mathbf{U})$ is extremely stiff. In fact, the numerical computation of the Jacobian matrix $\partial \mathbf{F} / \partial \mathbf{U}$ near a stable stationary value $\mathbf{F}(\mathbf{U})=0$, gives negative eigenvalues with negligible imaginary parts but with real parts going from $-10^{8}$ to about -1 . Moreover, it is impossible to separate fast and slow variables and even if this was possible, the voltage dependent gates would make this separation very local in time and space. All of these complex and stiff mechanisms yield a "detailed chemistry" description of the phenomenon. The explicit formulation of $\mathbf{F}(\mathbf{U})$ can be found in [10]. 


\subsection{D Configuration}

We first consider a computational domain of $[0,50000]^{2}(\mu \mathrm{m})$ and simulate the phenomenon over one hour $t \in[0,3600]$ (s). In what follows, we will refer to three ways to solve (2): the quasi-exact resolution, which considers the coupled reaction-diffusion problem (2) on a uniform mesh, computed by Radau5 with very fine tolerances; the splitting resolution, which uses the RDR Strang $\mathcal{S}_{2}^{\Delta t}$ scheme with Radau5 for the time integration of the reaction term and ROCK4 for the diffusive part, also on a uniform grid; and the proposed MR/splitting strategy, with the same $\mathcal{S}_{2}^{\Delta t}$ time integration scheme on an adapted mesh. All the computations have been performed on a 8 core $(2 \times 4) 64$ bits machine (AMD Shanghai processors) of $2.7 \mathrm{GHz}$ with memory capacity of 32 GB.
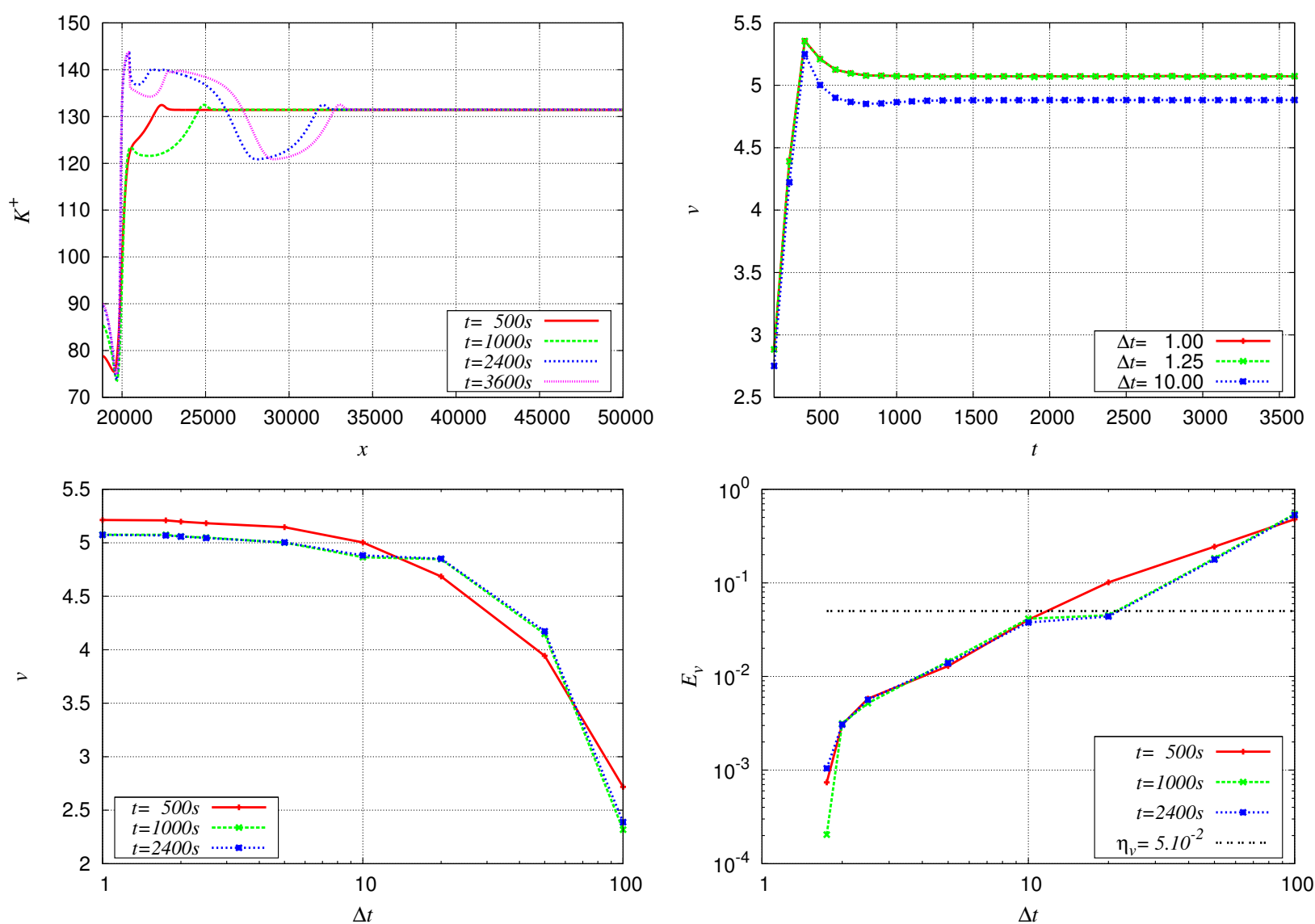

Figure 1. Top: time evolution of $K^{+}$in the neurons along $x$-axis (left) and of the wave velocity $v$ (right) for different splitting time steps $\Delta t$, computed on a uniform grid of 1024 points. Bottom: wave velocity $v$ (left) and velocity error $E_{v}$ computed by (8) at different times $t$ for different splitting time steps $\Delta t$.

Since the resolution of the coupled problem, the quasi-exact solution, with Radau5 is very expensive and we do not dispose any theoretical information on the solution of the problem, a preliminary study is required to choose an appropriate splitting time step according to $\S 1.4$. Therefore, we perform $1 \mathrm{D}$ computations with the splitting solver on a uniform grid of 1024 points in order to define a reference wave velocity $v$ into (8). Figure 1 (top) shows the propagation of the wavefront along the $x$-axis across the core of the initial perturbation (see 


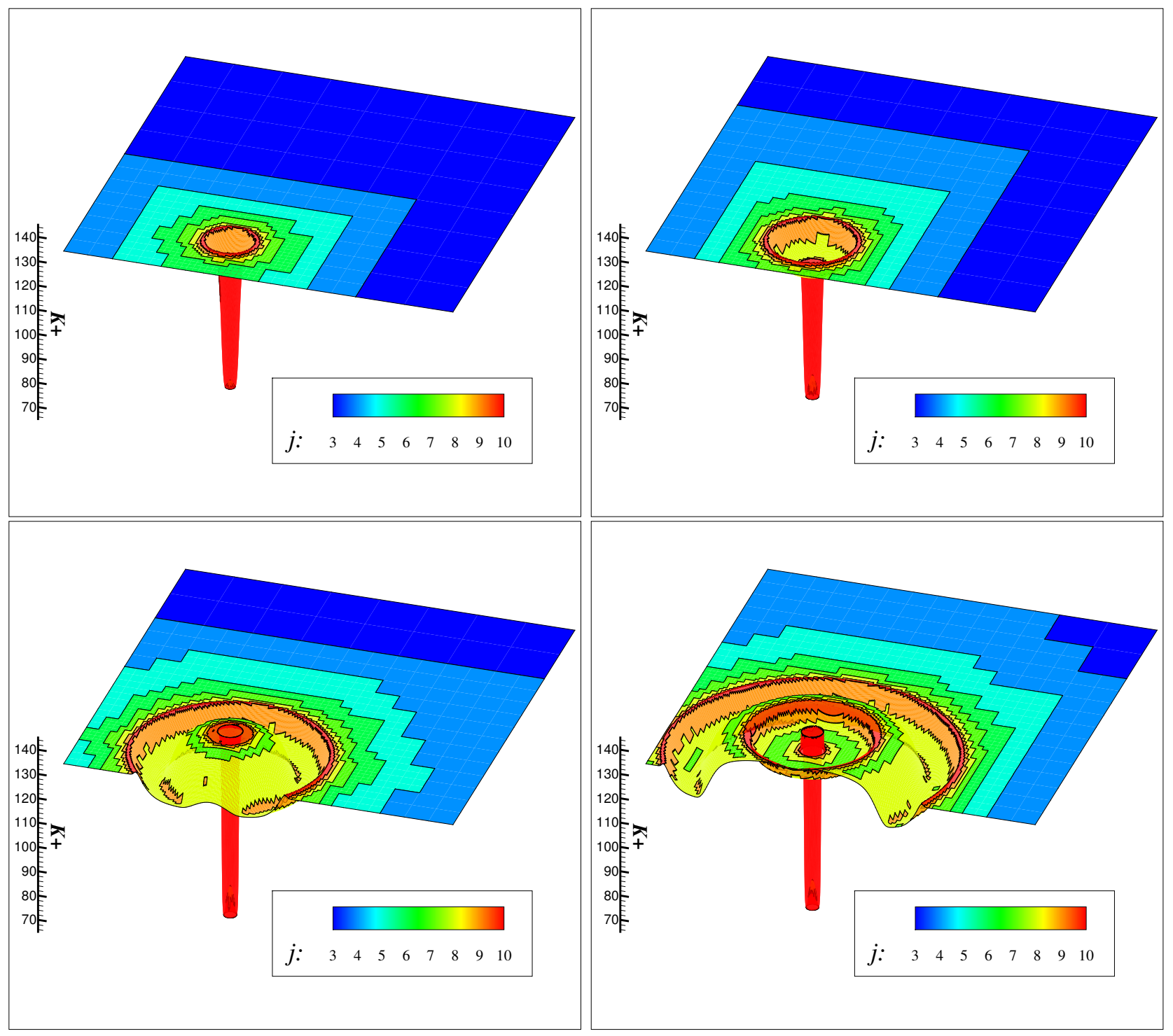

Figure $2 . K^{+}$in the neurons at $500 \mathrm{~s}$ (top left), $1000 \mathrm{~s}$ (top right), $2400 \mathrm{~s}$ (bottom left) and $3600 \mathrm{~s}$ (bottom right) represented on a dynamic adapted grid corresponding to $1024^{2}$ points at the finest level $\mathrm{J}=10$.

Figure 2), and the time evolution of the wave velocities for solutions computed with different splitting time steps. Smaller splitting time steps imply naturally a more accurate description of the global phenomenon, measured in this case by means of the wavefront speed (Figure 1 bottom left), and show convergence towards a roughly constant value of $v \approx 5.07$ once the propagating front is fully developed $(t \gtrsim 700 s)$ and the wave speed becomes relatively constant (Figure 1 top right). We take this velocity computed with $\Delta t=1$ as the reference one and choose a rather large splitting time step $\Delta t=10$ in order to show the potential decoupling of time steps for reaction, diffusion and the time operator splitting needed to solve the problem within a prescribed tolerance. This time step yields a relative velocity error of $\approx 3.8 \%$ (Figure 1 bottom right) or equivalently an accuracy tolerance of $\eta_{v}=5 \cdot 10^{-2}$ into (8). According to (10), this time step is bounded by the maximum splitting time 
step given by $\Delta t_{\max }=28.9$ for $\Delta x=\Delta y=50000 / 1024$ and $\hat{k}=3$ as in [11]. Tolerances of the solvers were set to $\eta_{\text {Radau } 5}=10^{-5}$ and $\eta_{R O C K 4}=10^{-7}$, after numerical experiments.

In order to analyze the performance of the $M R /$ splitting strategy proposed in [11], we consider several values of $\mathrm{J}$, that is the number of nested dyadic grids which gives a corresponding finest space resolution equivalent to $256^{2}(\mathrm{~J}=8), 512^{2}(\mathrm{~J}=9), 1024^{2}(\mathrm{~J}=10)$, or $2048^{2}(\mathrm{~J}=11)$ grid points. For all the simulations, we have considered a multiresolution threshold value of $\varepsilon=10^{-2}$ which yields a normalized $L^{2}$-error $\lesssim 10^{-2}$ between splitting and $M R /$ splitting resolutions for all 19 variables as shown in [12]. Higher spatial discretizations yield naturally better resolution of both the wave velocity and the dynamics of the wavefront. In particular, we have shown in [12] that at least $1024^{2}$ points are needed in order to get a reasonably fine description of the phenomenon. Figure 2 shows some $M R /$ splitting results for the time evolution of the concentration of $K^{+}$on the corresponding adapted grids, for an equivalent finest grid of $1024^{2}$ points $(\mathrm{J}=10)$.

Figure 3 shows the time evolution of the achieved data compressions (DC), and the number of points in the adapted grids (AG). DC is given by

$$
\mathrm{DC}=\left(1-\frac{\mathrm{AG}}{\mathrm{FG}}\right) \times 100
$$

where FG corresponds to the equivalent finest uniform grid $J$ in terms of spatial resolution. Data compression increases with the number of levels as the space scales present in the problem are better discriminated by finer spatial resolutions. For a given J, the number of points in the adapted grid AG is increasing linearly in time according to the growing radius with constant speed of the circular propagation of the wavefront as seen in Figure 2. On the other hand, at a given time, adding a new grid layer implies practically doubling the number of cells on the adapted grid as if we are only refining in one dimension as a consequence also of the quasi axial symmetry of the phenomenon.
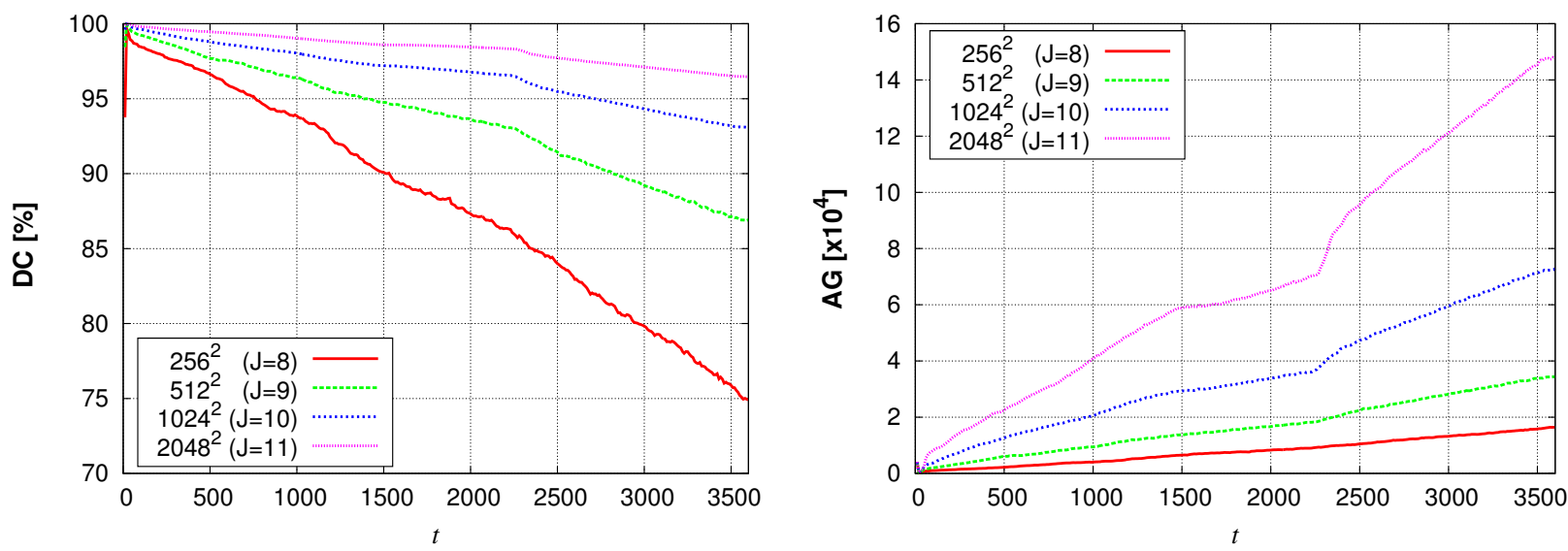

Figure 3. Time evolution of data compressions DC given by (11) (left) and the number of points on the adapted grids AG (right) for different finest levels J.

In order to take into account the memory requirements of each resolution strategy for a fine spatial resolution of $1024^{2}$, we estimate the array size of the working space needed by Radau5 and ROCK4:

(1) Radau5: $L_{1}=4 \times W_{1} \times W_{1}+12 \times W_{1}+20$ (from [13]);

(2) ROCK4: $L_{2}=8 \times W_{2}$ (from [1]);

where $W_{1}$ and $W_{2}$ are the number of unknowns solved by Radau5 and ROCK4. In the case of a uniform mesh, the total number of unknowns is $W=19 \times 1024^{2} \approx 1.99 \times 10^{7}$ and thus, the global size $L$ required for each solver is:

(1) Quasi-exact: $W_{1}=W \approx 1.99 \times 10^{7}$ and $L=L_{1} \approx 1.6 \times 10^{15}$. 
(2) Splitting: $W_{1}=19, W_{2}=10 \times W / 19 \approx 1.05 \times 10^{7}$ and $L=L_{1}+L_{2} \approx 8.4 \times 10^{7}$.

(3) $M R /$ Splitting with $\varepsilon=10^{-2}: W_{1}=19, W_{2}=0.07 \times 10 \times W / 19 \approx 7.34 \times 10^{5}$ and $L=L_{1}+L_{2} \approx 5.9 \times 10^{6}$; with minimum data compression of $93 \%$.

Considering a standard platform on which each double precision value is represented by 64 bits, each solver shall require $90.9 \mathrm{~Pb}, 5.0 \mathrm{~Gb}$ and $360.1 \mathrm{Mb}$. For standard computational resources, an implicit resolution with Radau5 is completely out of reach. These expensive memory requirements are strongly reduced with a splitting strategy but further reductions are achieved by adding a multiresolution adaptive procedure.

TABLE 1. Computing times (CT) in minutes and gain of parallelization (GP), for several finest grids (FG).

\begin{tabular}{|c|c|c|c|c|}
\hline \hline FG & $256^{2}$ & $512^{2}$ & $1024^{2}$ & $2048^{2}$ \\
\hline \hline CT & 10.92 & 31.37 & 82.45 & 214.43 \\
\hline GP & 7.62 & 7.50 & 7.55 & 7.52 \\
\hline \hline
\end{tabular}

Table 1 summarizes the computing times (CT) of the simulations, performed with 8 cores in parallel, and the gain of parallelization (GP) which is defined as the ratio between the computing time given by one single processor and the 8 cores in parallel. We see a rather high GP $(\max G P=8)$ even though only the time integration procedure is parallelized. Actually, for this kind of highly localized and stiff problems the multiresolution operations take normally less than $5 \%$ of the total time consumption. Parallelization of the reaction is practically optimal in the context of shared memory architectures because each core takes a new node immediately after finishing the previous one, without any need of synchronizing or exchanging data with the other cores.
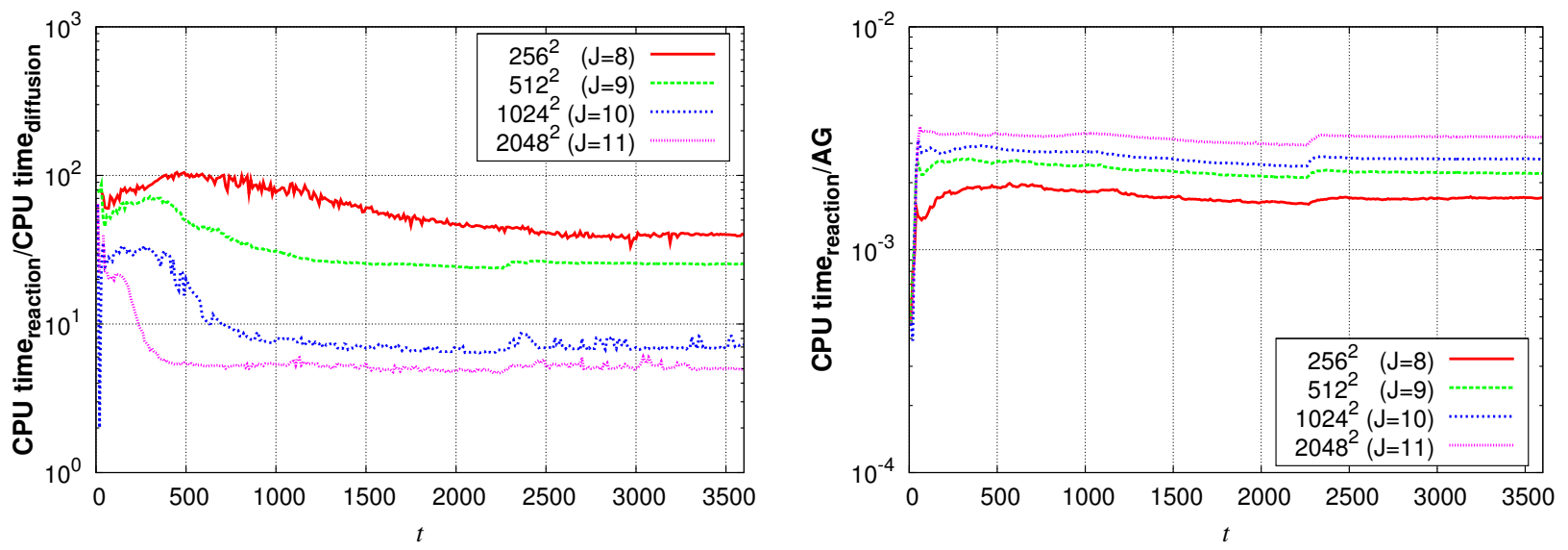

FiguRE 4. Time evolution of the ratio between CPU times for the integration of the reaction and diffusion problems (left) and the CPU time per number of points in the adapted grid for the reaction solution (right) for different finest levels J.

The integration of the stiff reaction problem is always much more expensive than any other computation in terms of CPU time. Figure 4 (left) shows the ratios in time between the CPU times used to integrate the reaction and the diffusion problems for different spatial discretizations. Whenever we add a new layer grid to the computational domain, the reaction CPU integration time per number of active points remains almost the same as seen in Figure 4 (right) because most of the new points will be naturally added at the wavefront where high chemical activity is present. Nevertheless, there is finally an overhead in CPU time for two successive finest grid configurations $\mathrm{J}$ as seen in Table 1 since we can measure an increase ratio of $\sim 2.6$, slightly higher 
than the ratio of 2 that we should have obtained taking into account the corresponding increase ratio of the adapted grids. Actually, as shown in Figure 4, the integration of the diffusion problem becomes more expensive when increasing the spatial discretization from $256^{2}$ to $2048^{2}$ because the diffusion time step $\Delta t_{D}$ dynamically chosen to guarantee the tolerance accuracy $\eta_{R O C K 4}$ goes respectively from $\sim 1.25$ to $\sim 0.27$. Furthermore, the matrix-vector products computed by ROCK4 also become more expensive since the matrix representation of the discretized diffusion operator is less sparse when the computational domain includes more grid levels. This is because in this multiresolution implementation we locally reconstruct the values at the interfaces of grids with different discretization in order to guarantee more accurate computations (see more details in [11]).
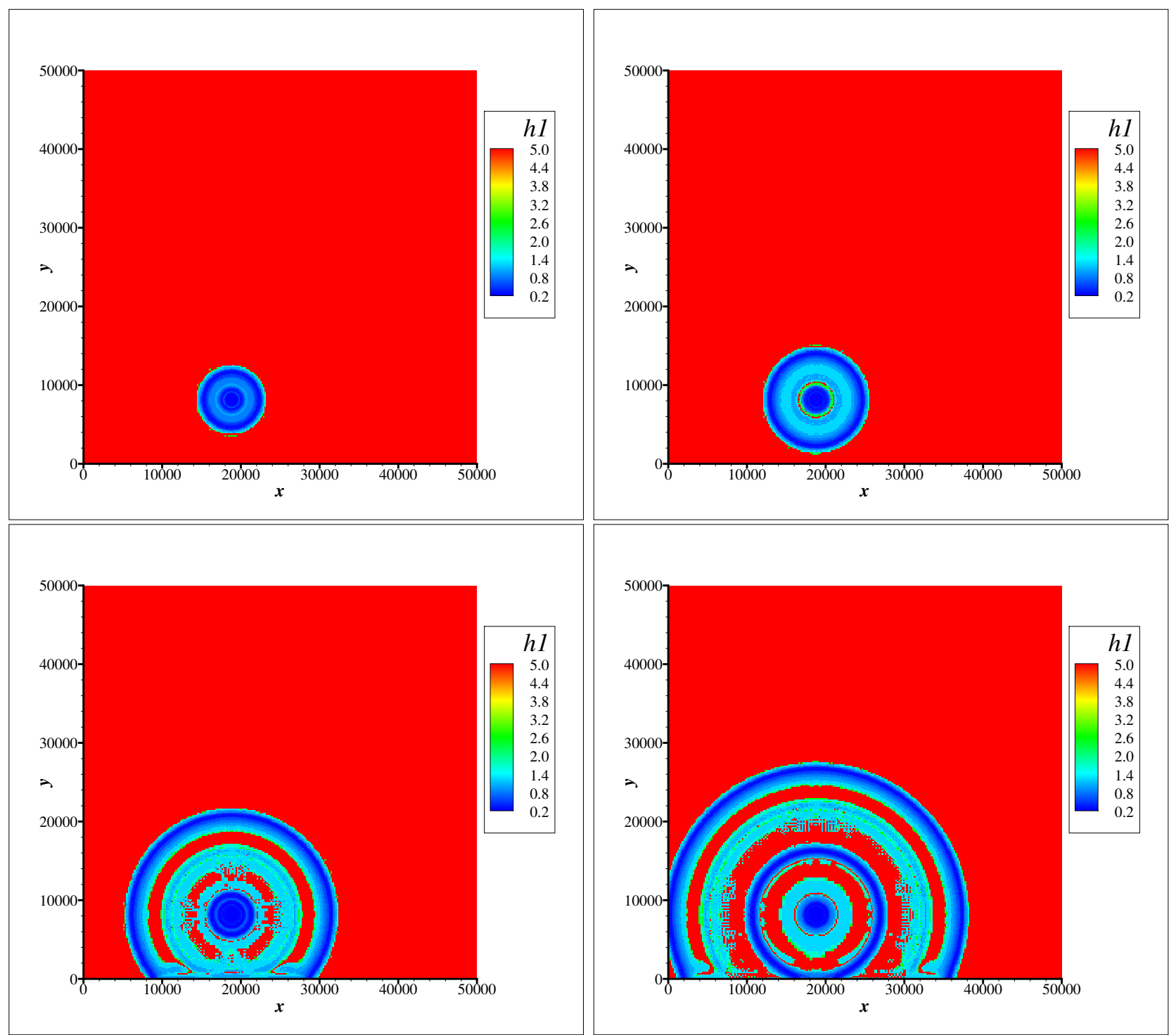

Figure 5. Local reaction time steps $h 1$ at $500 \mathrm{~s}$ (top left), $1000 \mathrm{~s}$ (top right), $2400 \mathrm{~s}$ (bottom left) and $3600 \mathrm{~s}$ (bottom right) within a half-splitting time step $\Delta t / 2=5$, represented on a dynamic adapted grid corresponding to $1024^{2}$ points at the finest level $\mathrm{J}=10$. 
The values of $\Delta t_{D}$ for different $\mathrm{J}$ imply 8 to 36 diffusion time steps within the splitting time step $\Delta t=10$ for which mainly the minimum $s=5$ stages are needed to guarantee the stability of the method according to (7). On the other hand, Figure 5 shows the corresponding local reaction time steps averaged within $\Delta t / 2$ for each point. The imposed tolerance for the reaction resolution $\eta_{\text {Radau } 5}$ implies time steps varying from $\sim 0.2$ to $\Delta t / 2=5$ for points located respectively in the neighborhood of the reactive fronts and the reduced chemical activity regions. Considering the refined regions, most of the computational domain uses reaction time steps 25 time larger than the smallest time steps for the prescribed tolerance. In this way, an effective decoupling of the numerical time scale spectrum is achieved for the reaction, the diffusion and the splitting schemes with an important gain of efficiency.

\subsection{D Configuration}

Let us consider now a 3D configuration with the same parameters as in the previous 2D case, in a space region of $[0,50000]^{3}(\mu \mathrm{m})$. In order to explore the feasibility and potential advantages of the method, we consider two cases with $\mathrm{J}=8$ and $\mathrm{J}=9$ nested dyadic grids, corresponding to $256^{3}$ and $512^{3}$ points in the finest grid J. For the first case of $256^{3}$, the achieved data compression DC amounts to $98.84 \%, 95.27 \%$ and $87.23 \%$ at times $1080 \mathrm{~s}, 2160 \mathrm{~s}$ and $3600 \mathrm{~s}$, respectively. The computing time CT was of about 16.68 hours with a gain of parallelization GP of 7.02 .

Figure 6 shows the concentration of $K^{+}$in the neurons and the corresponding adapted grids at $1080 \mathrm{~s}$ $(\mathrm{DC}=99.14 \%)$, and $2520 \mathrm{~s}(\mathrm{DC}=94.94 \%)$ for the $512^{3}$ case; $\mathrm{CT} \approx 45.88$ hours for $t \in[0,2520](\mathrm{s})$ and $\mathrm{GP}=7.14$. Longer simulations times yield larger simulation domains which are not longer feasible with the considered computing resource at least in a shared memory environment, and the current state of development of the code.

Performing the same comparison concerning memory requirements, the total number of unknowns for the second case is $W=19 \times 512^{3} \approx 2.55 \times 10^{9}$ and the global size of $L$ required by each solver is:

(1) Quasi-exact: $W_{1}=W \approx 2.55 \times 10^{9}$ and $L=L_{1} \approx 2.6 \times 10^{19}$.

(2) Splitting: $W_{1}=19, W_{2}=10 \times W / 19 \approx 1.34 \times 10^{9}$ and $L=L_{1}+L_{2} \approx 1.1 \times 10^{10}$.

(3) $M R /$ Splitting with $\varepsilon=10^{-2}: W_{1}=19, W_{2}=0.04 \times 10 \times W / 19 \approx 5.37 \times 10^{7}$ and $L=L_{1}+L_{2} \approx 4.3 \times 10^{8}$; with minimum data compression of $96 \%$.

Therefore, each solver shall require at least $1.4 \mathrm{Zb}, 655.7 \mathrm{~Gb}$ and $25.6 \mathrm{~Gb}$ of memory capacity.

\section{Conclusions}

In this paper, we implement a new numerical strategy recently introduced in [11] for the simulation of 2D and 3D multi-scale waves issued from a model of human ischemic stroke [10]. Based on recent mathematical studies $[7,9]$ that allow to better characterize the behavior of splitting techniques for time-space stiff PDEs and in particular for splitting time steps much larger than the fastest scales present in the problem, the proposed time operator splitting approach exploits these results with an important gain of efficiency regardless the large stiffness of the modeling equations. In the context of self-similar propagating waves, a constant splitting time step is enough to capture the dynamics of the phenomenon and it is computed based on some error estimates that allow to approximate the wave propagation velocity within a prescribed accuracy. On the other hand, dedicated high order time integration methods are chosen for the reaction and diffusion problems to properly solve the entire spectrum of temporal scales associated with each independent problem. In this way, an effective decoupling of the time scale spectrum is achieved whenever this is allowed by the physics of the problem, so that different physical or numerical time scales associated with each problem can be isolated and treated independently by each numerical method.

Coupling this time integration technique with an adaptive mesh refinement technique based on spatial multiresolution [5,15] yields highly compressed data representations that greatly improve the performance of the numerical strategy where the errors introduced by the compressed spatial representation can be controlled relying on the mathematical background of multiresolution analysis. In this way, for a problem represented with a 


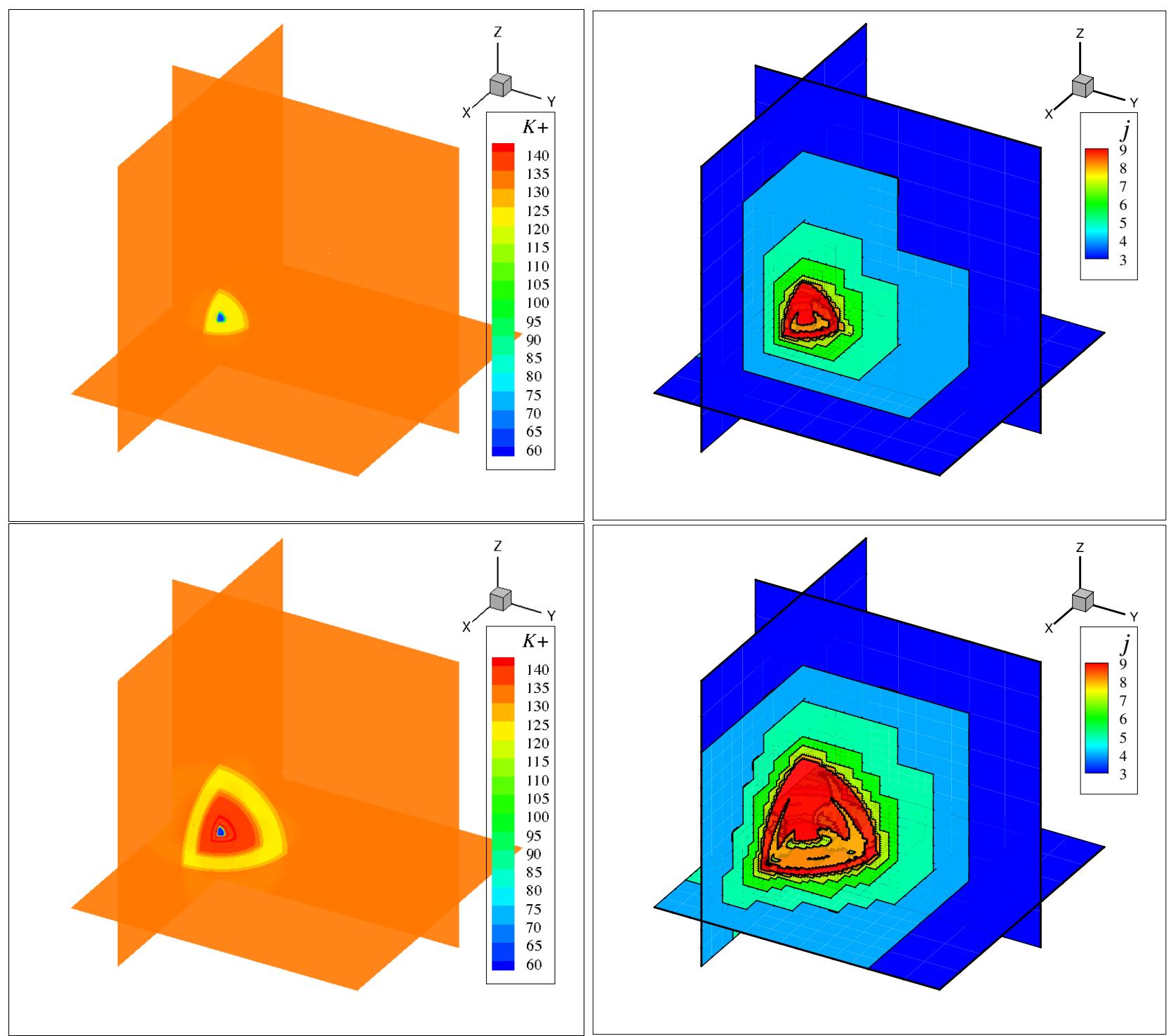

FIgURE $6 . K^{+}$in the neurons (left) and dynamic adapted grids (right) corresponding to $512^{3}$ points at the finest level $\mathrm{J}=9$ at $1000 \mathrm{~s}$ (top) and $2000 \mathrm{~s}$ (bottom).

spatial discretization limited mainly by the computational resources, the proposed $M R /$ splitting strategy allows to track the corresponding numerical error of the simulation introduced by the numerical methods of resolution. As a consequence, these highly stiff phenomena modeling complex chemical mechanisms previously out of reach, can be successfully simulated over large computational domains with conventional computing resources.

A straightforward parallelization technique was implemented for the time integration procedure that shows to be very efficient in the context of shared memory machines mainly because the global CPU time is largely dominated by the time resolution of the stiff reactive term. Nevertheless, an important amount of work is still in progress concerning programming features such as data structures, optimized routines and parallelization strategies for the time integration technique as well as the multiresolution environment in order to yet improve the performance of the method and to eventually obtain high efficiency in terms of load balancing on distributed 
parallel architectures for the simulation of such complex problems. For instance, some dedicated and efficient implementations have been recently developed for multiresolution applications $[2,3]$.

\section{REFERENCES}

[1] A. Abdulle. Fourth order Chebyshev methods with recurrence relation. SIAM J. Sci. Comput., 23:2041-2054, 2002.

[2] K. Brix, R. Massjung, and A. Voss. A hash data structure for adaptive PDE-solvers based on discontinuous Galerkin discretizations. IGPM-Rep. 302, RWTH Aachen, 2009.

[3] K. Brix, S. Melian, S. Müller, and G. Schieffer. Parallelisation of multiscale-based grid adaptation using space-filling curves. ESAIM: Proc., 29:108-129, 2009.

[4] A. Cohen. Wavelet methods in numerical analysis, volume 7. Elsevier, Amsterdam, 2000.

[5] A. Cohen, S.M. Kaber, S. Müller, and M. Postel. Fully adaptive multiresolution finite volume schemes for conservation laws. Math. of Comp., 72:183-225, 2003.

[6] Y. D'Angelo. Analyse et Simulation Numérique de Phénomènes liés à la Combustion Supersonique. PhD thesis, Ecole Nationale des Ponts et Chaussées, 1994.

[7] S. Descombes, T. Dumont, V. Louvet, and M. Massot. On the local and global errors of splitting approximations of reactiondiffusion equations with high spatial gradients. Int. J. of Computer Mathematics, 84(6):749-765, 2007.

[8] S. Descombes, T. Dumont, V. Louvet, M. Massot, F. Laurent, and J. Beaulaurier. Operator splitting techniques for multi-scale reacting waves and application to Low Mach number flames with complex chemistry: Theoretical and numerical aspects. In preparation, 2011.

[9] S. Descombes and M. Massot. Operator splitting for nonlinear reaction-diffusion systems with an entropic structure: Singular perturbation and order reduction. Numer. Math., 97(4):667-698, 2004.

[10] M.-A. Dronne, J.-P. Boissel, and E. Grenier. A mathematical model of ion movements in grey matter during a stroke. J. of Theoretical Biology, 240(4):599-615, 2006.

[11] M. Duarte, M. Massot, S. Descombes, C. Tenaud, T. Dumont, V. Louvet, and F. Laurent. New resolution strategy for multiscale reaction waves using time operator splitting, space adaptive multiresolution and dedicated high order implicit/explicit time integrators. Accepted to SIAM J. Sci. Comput, 2011. available on HAL (http://hal.archives-ouvertes.fr/hal-00457731).

[12] T. Dumont, M. Duarte, S. Descombes, M.-A. Dronne, M. Massot, and V. Louvet. Simulation of human ischemic stroke in realistic 3D geometry: A numerical strategy. Submitted to Bulletin of Math. Biology, 2011. available on HAL (http://hal.archivesouvertes.fr/hal-00546223).

[13] E. Hairer and G. Wanner. Solving ordinary differential equations II. Springer-Verlag, Berlin, second edition, 1996. Stiff and differential-algebraic problems.

[14] A. Harten. Multiresolution algorithms for the numerical solution of hyperbolic conservation laws. Comm. Pure and Applied Math., 48:1305-1342, 1995.

[15] S. Müller. Adaptive multiscale schemes for conservation laws, volume 27. Springer-Verlag, Heidelberg, 2003.

[16] L. F. Shampine, B. P. Sommeijer, and J. G. Verwer. IRKC: An IMEX solver for stiff diffusion-reaction PDEs. J. Comput. Appl. Math., 196(2):485-497, 2006.

[17] B. Sportisse. An analysis of operator splitting techniques in the stiff case. J. Comput. Phys., 161(1):140-168, 2000.

[18] G. Strang. On the construction and comparison of difference schemes. SIAM J. Numer. Anal., 5:506-517, 1968.

[19] J. G. Verwer. Explicit Runge-Kutta methods for parabolic partial differential equations. Appl. Numer. Math., 22(1-3):359-379, 1996.

[20] J. G. Verwer, B. P. Sommeijer, and W. Hundsdorfer. RKC time-stepping for advection-diffusion-reaction problems. J. Comput. Phys., 201(1):61-79, 2004.

[21] J. G. Verwer and B. Sportisse. Note on operator splitting in a stiff linear case. Rep. MAS-R9830, 1998. 